Steering Committee, which McCormick co-chairs, fears CIHR will dismantle the Institute of Aboriginal Peoples' Health altogether, although the external working group report notes its members "do not have sufficient knowledge to judge whether any Institute should be dismantled or merged."

The committee's request for a consultation before CHIR implements any changes has not yet been granted. In a Dec. 9 letter to Wien, Beaudet confirmed many of the changes but said two vicepresidents and the scientific director of the Institute of Aboriginal Peoples' Health will "reach out to the community to establish meaningful dialogue" and strengthen Aboriginal health-research capacity.

Several First Nations researchers are particularly concerned about the effect of cutting funding for students.

"Our prospects for continuing the journey to culturally appropriate research equity is wiped away," Dr. Judith Bartlett, a Métis associate professor at the University of Manitoba, wrote in an open letter to Beaudet Nov. 18. - Laura Eggertson, Ottawa, Ont.

CMAJ 2015. DOI:10.1503/cmaj.109-4965

\title{
Bruce Squires Award for microbiome study
}

$\mathrm{T}$ he authors of a study looking at the variability of gut flora in healthy infants with different diet and delivery methods have been awarded for their contribution to health care research in Canada.

Dr. Meghan Azad and coauthors are the recipients of the Bruce Squires Award for their article "Gut microbiota of healthy Canadian infants: profiles by mode of delivery and infant diet at 4 months" (CMAJ 2013;185:385-94). It was one of the most highly cited CMAJ articles in 2014 and had significant downloads.

The Bruce Squires Award is presented annually to the authors of a research article that promotes high standards of reporting and evidence-based medicine. The award honours the late Dr. Bruce Squires who was CMAJ's editor-in-chief from 1989-1996.

"The Bruce Squires Award is given for research that is likely to have a high impact on patient care, so we like to choose research that is either at the beginning of a research story or at the end of a research story," said CMAJ Editor-inChief Dr. John Fletcher. "In previous years we have awarded systematic reviews giving the definitive result at the end of a story of research. This year's winner is a break with tradition in that it is at the beginning of the story of the microbiome and how our gut flora interacts with our immune system and produces disease." Fletcher said he has high hopes for this area of research in the future.

"Gut microbes clearly play an important role in human health and disease, but microbiome research is a relatively new field, and most studies have been conducted in adults," said Azad. The CMAJ

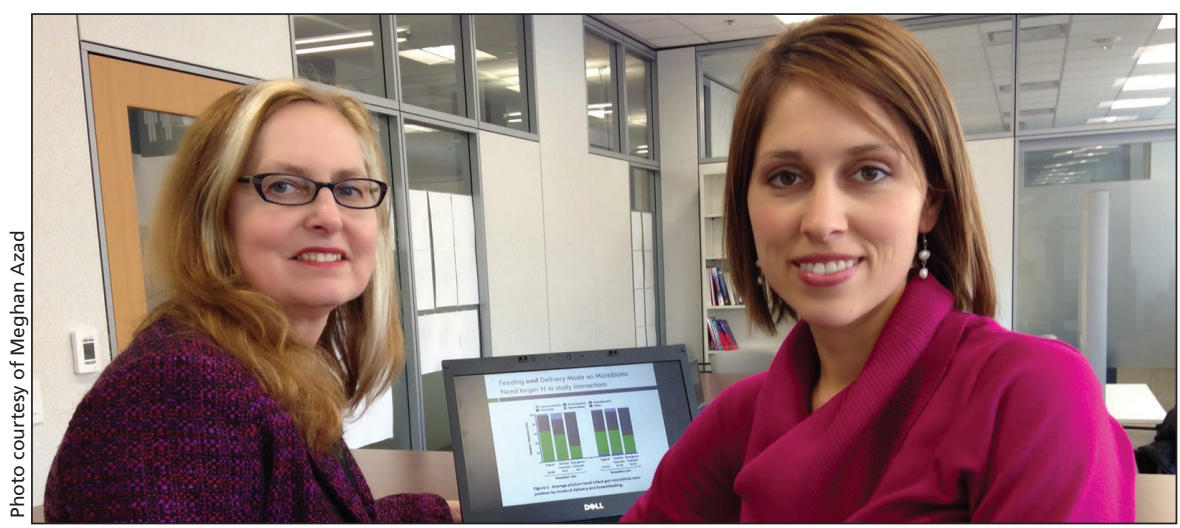

Dr. Anita Kozyrskyj (left) and Dr. Meghan Azad are members of the research team that received the Bruce Squires Award for their study on the gut microbiome of infants.

study examined the microbiome of 24 infants who were recruited as part of the Canadian Healthy Infant Longitudinal Development (CHILD) cohort, which was conducted at four sites. Researchers studied the changes in gut flora based on delivery mode, use of maternal antibiotics, group B streptococcus status and diet. The authors found that infant gut flora varied by delivery and breastfeeding status. Bacteroides was underrepresented in infants born through cesarean delivery when compared to infants delivered vaginally. In addition, the diversity of gut microbiota in breastfed infants was lower than that of formula-fed infants. These findings are consistent with those in previous studies in Europe, but used new DNA sequencing technology to identify a greater diversity of gut flora.

Azad, an assistant professor of Pediatrics and Child Health at the University of Manitoba in Winnipeg, completed her postdoctoral fellowship in pediatrics at the University of Alberta in Edmonton. Her research focuses on the gut microbiome and the early-life origins of chronic disease. She coauthored the paper with the primary investigators of the Synergy in Microbiota team: Dr. Anita Kozyrskyj, professor of pediatrics at the University of Alberta and Dr. James Scott, associate professor, Dalla Lana School of Public Health, University of Toronto.

"My team and I are absolutely delighted to receive the award for our research which represents the first microbiome study of North American infants," said Kozyrskyj. "We intentionally selected $C M A J$ because of our interest in evaluating early life interventions such as cesarean section delivery and antibiotic treatment as being very relevant to obstetricians, pediatricians and of course parents."

The team is expanding the study to include more infants and long-term follow up. Infant feeding and birth practices are complex decisions for parents. The research identifies gut microbiota as a new factor to consider in this process, said Kozyrskyj. — Moneeza Walji, CMAJ

CMAJ 2015. DOI:10.1503/cmaj.109-4970 